\title{
OSCILLATORY BEHAVIOR OF SECOND ORDER UNSTABLE TYPE NEUTRAL DIFFERENCE EQUATIONS
}

\author{
E. THANDAPANI, S. PANDIAN AND R. K. BALASUBRAMANIAN
}

\begin{abstract}
This paper deals with the oscillatory behavior of all bounded/ unbounded solutions of second order neutral type difference equation of the form

$$
\left.\Delta\left(a_{n}\left(\Delta_{c} y_{n}+p y_{n-k}\right)\right)^{\alpha}\right)-g_{n} f\left(y_{\sigma(n)}\right)=0
$$

where $p$ is real, $\alpha$ is a ratio of odd positive integers, $k$ is a positive integer and $\{\sigma(n)\}$ is a sequence of integers. Examples are provided to illustrate the results.
\end{abstract}

\section{Introduction}

Consider the difference equation of the form

$$
\left.\left.\Delta a_{n}\left(\Delta_{c} y_{n}+p y_{n-k}\right)\right)^{\alpha}\right)-g_{n} f\left(y_{\sigma(n)}\right)=0
$$

where $n \in \mathbb{N}\left(n_{0}\right)=\left\{n_{0}, n_{0}+1 \cdots\right\}, n_{0}$ a nonnegative integer, $\Delta$ is the forward difference operator defined by $\Delta y_{n}=y_{n+1}-y_{n}$, subject to the conditions:

$\left(c_{1}\right) p$ is a real number, $k$ is a positive integer and $\alpha$ is a ratio of odd positive integers;

$\left(c_{2}\right)\left\{a_{n}\right\}$ is a positive sequence such that $\sum_{n=n_{0}}^{\infty} \frac{1}{a_{n}^{1 / \alpha}}=\infty$ and $\left\{g_{n}\right\}$ is a non negative sequence of real numbers and $g_{n}$ is not identically zero for infinitely many values of $n$;

$\left(c_{3}\right)\{\sigma(n)\}$ is a sequence of integers such that $\sigma(n) \rightarrow \infty$ as $n \rightarrow \infty$;

$\left(\mathrm{c}_{4}\right) \quad f: \mathbb{R} \rightarrow \mathbb{R}$ is a continuous function with $u f(u)>0$ and $\frac{f(u)}{u^{\alpha}} \geq M>0$ for $u / 0$.

Let $\theta=\max \{k, \inf \sigma(n)\}$. By a solution of equation (1), we mean a real sequence $\left\{y_{n}\right\}$ defined for $n \geq n_{0}-\theta$ and satisfying equation (1) for $n \in \mathbb{N}\left(n_{0}\right)$ and $\sup \left\{\left|y_{s}\right|: s \geq n\right\}>0$ for $n \in \mathbb{N}\left(n_{0}\right)$. Such a solution $\left\{y_{n}\right\}$ is called oscillatory if for any $n_{1} \in \mathbb{N}\left(n_{0}\right)$, there are integers $n_{2}, n_{3} \geq n_{1}$ such that $y_{n_{2}} y_{n_{3}} \leq 0$ and is called nonoscillatory otherwise.

Equations of this type arise in a number of important applications such as problems in population dynamics when maturation and gestation are included, in cobweb models in economics where demand depends on current price but supply depends on the price

Received September 8, 2003; revised October 28, 2003.

2000 Mathematics Subject Classification. 39A10.

Key words and phrases. Neutral difference equation, bounded solution, unbounded solution, oscillation. 
at an earlier time, and in electric networks containing lossless transmission lines. Hence it is important and useful to study the oscillatory properties of solution of equation (1).

In most of the papers $[1,2,6,7,10,11]$, the authors established conditions for the oscillation and nonoscillation of solutions of equation of type (1) with $\alpha=1$ and treating the deviations are constant. In $[1,3,5,8,9,12]$, the authors consider the particular cases of equation (1) in the form

$$
\left.\Delta\left(a_{n}\left(\Delta y_{n}\right)^{\alpha}\right)-g_{n} f\left(y_{\alpha(n)}\right)\right)=0
$$

or

$$
\Delta\left(a_{n} \Delta y_{n}\right)-g_{n} f\left(y_{n+1}\right)=0
$$

and discussed the oscillatory and asymptotic behavior of solutions of equations (2) and (3).

Motivated by this observation in this paper our aim is to study the oscillatory behavior of solutions of equation (1) under the assumption that the deviation $\sigma(n)-n$ is not neccessarily constant and may be unbounded. In Section 2, we obtain conditions for the oscillation of all bounded solutions of equation (1) under the assumption that $\{\sigma(n)\}$ is increasing such that $\sigma(n) \leq n$ and in Section 3, we establish conditions for all unbounded solution of equation (1) to be oscillatory under the assumption that $\{\sigma(n)\}$ in increasing such that $\sigma(n)>n$. Examples are inserted to illustrate our results.

\section{Bounded Oscillation of Equation (1)}

From Theorem 3.1 of [6] it is clear that it is not possible to find criteria for all the solutions of equation (1) to be oscillatory when $\{\sigma(n)\}$ is increasing with $\sigma(n) \leq n$. However in this section we establish conditions for the oscillation of all bounded solutions of equation (1).

Theorem 1. With respect to the difference equation (1) assume that $-1<p \leq 0$. If

$$
\lim _{n \rightarrow \infty} \sup \sum_{s=\sigma(n)}^{n}\left(\frac{1}{a_{s}} \sum_{t=s}^{n} g_{t}\right)^{\frac{1}{\alpha}}>\frac{1}{M^{\frac{1}{\alpha}}}
$$

then every bounded solution of equation (1) is oscillatory.

Proof. Without loss of generality, we may assume that $\left\{y_{n}\right\}$ is an eventually positive solution of equation (1). Define

$$
z_{n}=y_{n}+p y_{n-k}
$$

From equation (1), we have $\Delta\left(a_{n}\left(\Delta z_{n}\right)^{\alpha}\right) \leq 0$ for all large $n \in \mathbb{N}\left(n_{0}\right)$. If $a_{n}\left(\Delta z_{n}\right)^{\alpha}>0$ eventually, then $\lim _{n \rightarrow \infty} z_{n}=\infty$, which contradicts the boundedness of $\left\{y_{n}\right\}$. Therefore $a_{n} \Delta z_{n}<0$ since $\alpha$ is a ratio of odd positive integers and $\left\{a_{n}\right\}$ is eventally positive we may take $\Delta z_{n}<0$ for $n \in \mathbb{N}\left(n_{0}\right)$. Now, we have two possibilities for $\left\{z_{n}\right\}$ : 
(a) $z_{n}>0$ for $n \geq n_{1} \in \mathbb{N}\left(n_{0}\right)$;

(b) $z_{n}<0$ for $n \geq n_{1}$.

In case (a), equation (1) can be written as

Using (5), we obtain

$$
\Delta\left(a_{n}\left(\Delta z_{n}\right)^{\alpha}\right) \geq M g_{n} y_{\sigma(n)}^{\alpha} .
$$

$$
\Delta\left(a_{n}\left(\Delta z_{n}\right)^{\alpha}\right) \geq M g_{n} z_{\sigma(n)}^{\alpha} .
$$

Summing (6) from $s$ to $n$ yields

$$
a_{n+1}\left(\Delta z_{n+1}\right)^{\alpha}-a_{s}\left(\Delta z_{s}\right)^{\alpha} \geq M \sum_{t=s}^{n} q_{t} z_{\sigma(t)}^{\alpha} .
$$

or

$$
-\Delta z_{s} \geq z_{\sigma(n)}\left(\frac{M}{a_{s}} \sum_{t=s}^{n} q_{t}\right)^{\frac{1}{\alpha}}
$$

Summing the last inequality in $s$ from $\sigma(n)$ to $n$, we see that

$$
-z_{n+1}+z_{\sigma(n)} \geq M^{\frac{1}{\alpha}} z_{\sigma(n)} \sum_{s=\sigma(n)}^{n}\left(\frac{1}{a_{s}} \sum_{t=s}^{n} q_{t}\right)^{\frac{1}{\alpha}} .
$$

Hence for $n \geq n_{1}$, we have,

$$
z_{n+1}+z_{\sigma(n)}\left(M^{\frac{1}{\alpha}} \sum_{s=\sigma(n)}^{n}\left(\frac{1}{a_{s}} \sum_{t=s}^{n} q_{t}\right)^{\frac{1}{n}}-1\right) \leq 0
$$

which contradiction (4). For the case (b), we obtain

$$
y_{n}<-p y_{n-k}<(-p)^{2} y_{n-2 k}<\cdots<(-p)^{j} y_{n-j k}
$$

for $n \geq n_{1}+j k$ and we are led to that $\lim _{n \rightarrow \infty} y_{n}=0$. Hence $\lim _{n \rightarrow \infty} z_{n}=0$. which is again a contradiction. This completes the proof of the theorem.

Remark 1. If $\alpha=1, a_{n} \equiv 1$ and $\sigma(n)=n-l$, then Theorem 1 reduces to Theorem 4.1 of Lalli and Zhang [6].

To prove our next result, we need the following lemma.

Lemma 1. Let $\left\{y_{n}\right\}$ be an eventually positive sequence and $z_{n}=y_{n}+p y_{n-k}$ be such that $\Delta z_{n}<0$ for all $n \in \mathbb{N}\left(n_{0}\right)$. Then there is an integer $N \in \mathbb{N}\left(n_{0}\right)$ such that

if $-1<p \leq 0$ and

$$
y_{n} \geq \frac{z_{n}}{1+p}, \quad n \geq N
$$




$$
y_{n} \geq \frac{z_{n+k}}{1+p}, \quad n \geq N
$$

if $p>0$.

Proof. Since $\left\{z_{n}\right\}$ is decreasing, from $z_{n}=y_{n}+p y_{n-k}$, we may assume without loss of generality that, $\left\{y_{n}\right\}$ is also decreasing for $n \geq N \in \mathbb{N}\left(n_{0}\right)$, (see [4]). Hence for $-1<p \leq 0$, we have

$$
z_{n}=y_{n}+p y_{n-k} \leq y_{n}+p y_{n}, \quad n \geq N
$$

and therefore $y_{n} \geq z_{n / 1+p}, n \geq N$. If $p>0$, then we have

$$
z_{n} \leq y_{n-k}+p y_{n-k}, \quad n \geq N
$$

and we have

$$
y_{n} \geq \frac{z_{n+k}}{1+p}, \quad n \geq N
$$

This completes the proof.

Theorem 2. With respect to the difference equation (1) assume that $-1<p \leq 0$. If

$$
\lim _{n \rightarrow \infty} \sup \sum_{s=\sigma(n)}^{n}\left(\frac{1}{a_{s}} \sum_{t=s}^{n} q_{t}\right)^{\frac{1}{\alpha}}>\frac{1+p}{M^{\frac{1}{\alpha}}}
$$

then every bounded solution of equation (1) is oscillatory.

Proof. Assume that $\left\{y_{n}\right\}$ is an eventually positive bounded solution of equation (1). We can proceed exactly as in the proof of Theorem 1, to see that there are two possibilities for $\left\{z_{n}\right\}$ :

(a) $z_{n}>0, \Delta z_{n}<0, \Delta\left(a_{n}\left(\Delta z_{n}\right)^{\alpha}\right) \geq 0$ for $n \geq n_{1} \in \mathbb{N}\left(n_{0}\right)$ :

(b) $z_{n}<0, \Delta z_{n}<0, \Delta\left(a_{n}\left(\Delta z_{n}\right)^{\alpha}\right) \geq 0$ for $n \geq n_{1}$.

In case (a), from equation (1), we have

$$
\Delta\left(a_{n}\left(\Delta z_{n}\right)^{\alpha}\right) \geq M g_{n} y_{\sigma(n)}^{\alpha}, \quad n \geq n_{1} .
$$

Now, using Lemma 1, we obtain

$$
\Delta\left(a_{n}\left(\Delta z_{n}\right)^{\alpha}\right) \geq \frac{M g_{n}}{(1+p)^{\alpha}} z_{\sigma(n)}^{\alpha}, \quad n \geq n_{1} .
$$

Proceeding now exactly as in the proof of Theorem 1, we obtain

$$
z_{n+1}+z_{\sigma(n)}\left(\frac{M^{\frac{1}{\alpha}}}{1+p} \sum_{s=\sigma(n)}^{n}\left(\frac{1}{a_{s}} \sum_{t=s}^{n} q_{t}\right)^{\frac{1}{a}}-1\right) \leq 0
$$


which contradicts condition (8). The case (b) can be handled similarly as in the proof of Theorem 1. The proof is now complete.

Remark 2. It is easy to see that the results obtained in Theorem 2 is better than that of Theorem 1 since the constant $p$ includes in condition (8). Further Theorem 2 improves and generalize Theorem 4.1 of Lalli and Zhang [6].

Remark 3. Theorem 2 is true for $p=0$. This is due to Wong and Agarwal [12]. Further, if $a_{n}=1 . \sigma(n)=n-1$ and $a_{n} \equiv 1$, then Theorem 2 reduces to Theorem 1 of Thandapani, Arul and Raja [11].

Example 1. Consider the difference equation

$$
\Delta\left(\left(\Delta\left(y_{n}-\frac{1}{2} y_{n-k}\right)\right)^{\alpha}\right)=c y_{n-l}^{\alpha}, \quad n \in \mathbb{N}\left(n_{0}\right)
$$

Here $p=-\frac{1}{2}, a_{n}=1, q_{n}=c, \sigma(n)=n-l$ and the condition (8) becomes

$$
\sum_{s=1}^{l+1} c^{\frac{1}{\alpha}} s^{\frac{1}{\alpha}}>\frac{1}{2}
$$

Thus, by Theorem 2 if (10) is satisfied then all bounded solutions of equation (9) are oscillatory. This is particularly so when $\alpha=3, l=2$ and $c>0.034$.

Theorem 3. With respect to the difference equation (1) assume that $p=-1$. If

$$
\lim _{n \rightarrow \infty} \sup \sum_{s=\sigma(n)}^{n}\left(\frac{1}{a_{s}} \sum_{t=s}^{n} q_{t}\right)^{\frac{1}{\alpha}}>\frac{1}{M^{\frac{1}{\alpha}}}
$$

then every bounded solution of equation (1) is oscillatory.

Proof. Assume that $\left\{y_{n}\right\}$ is an eventually positive solution of equation (1). Proceeding as in the proof of Theorem 1 , we see that there are two possibilities for $\left\{z_{n}\right\}$ :

(a) $z_{n}>0, \Delta z_{n}<0, \Delta\left(z_{n}\left(\Delta z_{n}\right)^{\alpha}\right) \geq 0, n \geq n_{1} \in \mathbb{N}\left(n_{0}\right)$;

(b) $z_{n}<0, \Delta z_{n}<0, \Delta\left(z_{n}\left(\Delta z_{n}\right)^{\alpha}\right) \geq 0, n \geq n_{1}$

In case (a), we are led to (7) which contradicts condition (11). In the case (b), we have $\lim _{n \rightarrow \infty} z_{n}=-\beta$ where $\beta>0$ is a finite number. So there is an integer $n_{2} \in \mathbb{N}\left(n_{0}\right)$ such that $-\beta<z_{n}<-\frac{\beta}{2}$ for $n \geq n_{2} \geq n_{1}$. Hence

$$
-\beta<y_{n}-y_{n-k}<-\frac{\beta}{2}, \quad n \geq n_{2} .
$$

Then,

$$
y_{n}-\frac{\beta}{2}+y_{n+k}<-2 \frac{\beta}{2}+y_{n-2 k}<\cdots<-j \frac{\beta}{2}+y_{n-j k}
$$


for $n \geq n_{2}+j k$. Choose a sequence $\left\{n_{j}\right\}$ such that $n_{i}=n_{2}+j k$. Then

$$
y_{n_{2}+j k}<j \frac{\beta}{2}+y_{n_{2}}
$$

and therefore $\lim _{j \rightarrow \infty} y_{n_{j}}=-\infty$. This is a contradiction to the boundedness of $\left\{y_{n}\right\}$. This completes the proof.

In the following, we present another oscillatory criterion for equation (1) where $p \leq$ -1 .

Theorem 4. With respect to the difference equation (1) assume that $p \leq-1$. If

$$
\sum_{n=n_{0}}^{\infty}\left(\frac{1}{a_{n}} \sum_{s=n}^{\infty} q_{s}\right)^{\frac{1}{\alpha}}=\infty
$$

then every bounded solution of equation (1) is oscillatory.

Proof. Assume without loss of generality that $\left\{y_{n}\right\}$ is a bounded positive solution of equation (1) and $z_{n}$ is defined by (5). Thus there are two possibilities for $\left\{z_{n}\right\}$ :

(a) $z_{n}>0, \Delta z_{n}<0$ and $\Delta\left(a_{n}\left(\Delta z_{n}\right)^{\alpha}\right) \geq 0, n \geq n_{1} \in \mathbb{N}\left(n_{0}\right)$;

(b) $z_{n}<0, \Delta z_{n}<0$ and $\Delta\left(a_{n}\left(\Delta z_{n}\right)^{\alpha}\right) \geq 0, n \geq n_{1}$.

In case (a), we have $y_{n}>-p y_{n-k}$ for $n \geq n_{1}$ and there exists a constant $\delta>0$ such that $y_{n} \geq \delta$ for $n \geq n_{1}$. Hence from equation (1), we have

$$
\Delta\left(a_{n}\left(\Delta z_{n}\right)^{\alpha}\right) \geq M \delta^{\alpha} q_{n}, \quad n \geq n_{1} .
$$

In case (b), there exists a finite number $\beta>0$ such that $\lim _{n \rightarrow \infty} z_{n}=-\beta$. Then there exists an integer $n_{2} \geq n_{1}$ such that $-\beta<z_{n}<-\frac{\beta}{2}$ for $n \geq n_{2}$, that is,

$$
-\beta<y_{n}+p y_{n-k}<-\frac{\beta}{2}
$$

for $n \geq n_{2}$. Hence $y_{n-k}>\frac{\beta}{2 p}, n \geq n_{2}$. Then there exists an integer $n_{3} \geq n_{2}$ such that $y_{\sigma(n)}>\frac{\beta}{2}, n \geq n_{3}$. From equation (1), we have

$$
\Delta\left(a_{n}\left(\Delta\left(z_{n}\right)^{\alpha}\right) \geq M\left(\frac{P}{2}\right)^{\alpha} q_{n}, \quad n \geq n_{3} .\right.
$$

Thus in both the cases we are led to the inequality

$$
\Delta\left(a_{n}\left(\Delta\left(z_{n}\right)^{\alpha}\right) \geq B q_{n}, \quad n \geq n_{3}\right.
$$

where $B$ is a constant. Summing (13) from $n$ to $N$ for $N>n \geq n_{3}$, we have

$$
a_{N+1}\left(\Delta z_{N+1}\right)^{\alpha}-a_{n}\left(\Delta z_{n}\right)^{\alpha} \geq B \sum_{s=n}^{N} q_{s}, \quad n_{3} \leq n<N .
$$


Hence

$$
-a_{n}\left(\Delta z_{n}\right)^{\alpha} \geq B \sum_{s=n}^{N} q_{s}, \quad n_{3} \leq n<N
$$

which implies that $\sum_{s=n_{0}}^{\infty} q_{s}<\infty$ and so

$$
-\Delta z_{n} \geq\left(\frac{B}{a_{n}} \sum_{s=n}^{\infty} q_{s}\right)^{\frac{1}{\alpha}} .
$$

Summing the last inequality from $n$ to $N-1$ for $N-1>n$, we have

or

$$
z_{n} \geq z_{N}+B^{\frac{1}{\alpha}} \sum_{s=n}^{N-1}\left(\frac{1}{a_{s}} \sum_{t=s}^{\infty} q_{t}\right)^{\frac{1}{\alpha}}
$$

$$
z_{n_{0}} \geq B^{\frac{1}{\alpha}} \sum_{s=n_{0}}^{\infty}\left(\frac{1}{a_{s}} \sum_{t=s}^{\infty} q_{t}\right)^{\frac{1}{\alpha}}
$$

which contradicts condition (12). This completes the proof.

Example 2. Consider the difference equation.

$$
\Delta\left(\left(\Delta\left(y_{n}-2 y_{n-k}\right)\right)^{3}\right)=\frac{128}{(n-4)^{3}} y_{n-5}^{3}, \quad n \geq 5 .
$$

It is easy to see that all assumptions of Theorem 4 are satisfied. Therefore, every bounded solution of equation (14) is oscillatory.

Remark 4. If $\alpha=1, a_{n} \equiv 1$ and $\sigma(n)=n-1$, then Theorem 4 reduces to Theorem 4.2 of Lalli and Zhang [6].

Our final result in this section deals with the case $p>0$.

Theorem 5. With respect to the difference equation (1), assume that $p>0$ and $\sigma(n)=n-l$ where $l$ is a positive integer such that $l \geq k+1$. If

$$
\lim _{n \rightarrow \infty} \sup \sum_{s=n+k-l}^{n}\left(\frac{1}{a_{s}} \sum_{t=s}^{n} q_{t}\right)^{\frac{1}{\alpha}}>\frac{1+p}{M^{\frac{1}{\alpha}}}
$$

then all bounded solutions of equation (1) are oscillatory.

Proof. The proof is similar to that of Theorem 2 and hence the details are omitted.

Example 3. Consider the difference equation

$$
\Delta\left(\left(\Delta\left(y_{n}+p y_{n+2}\right)\right)^{3}\right)+8(1+p)^{3} y_{n-4}^{3}\left(1+\left|y_{n-4}\right|\right)=0 .
$$


All conditions of Theorem 5 are satisfied and hence all bounded solutions of equation (15) are oscillatory. In fact; $\left\{y_{n}\right\}=\left\{(-1)^{n}\right\}$ is such a solution of equation (15).

\section{Unbounded Oscillation of Equation (1)}

In this section we present sufficient conditions for the oscillation of all unbounded solution of equation (1) when $\sigma(n)=n+l$, where $l$ is a positive integer.

Theorem 6. With respect to the difference equation (1) assume that

$$
0 \leq p<1
$$

and

$$
\lim _{n \rightarrow \infty} \sup \sum_{s=n}^{n+l-1}\left(\frac{1}{a_{s}} \sum_{t=n}^{s-1} q_{t}\right)^{\frac{1}{\alpha}}>M^{\frac{1}{\alpha}}(1-p)
$$

hold. Then every unbounded solution of equation (1) oscillates.

Proof. Assume for the sake of contradiction that equation (1) has an eventually positive unbounded solution $\left\{y_{n}\right\}$. Then $y_{n}>0$ for $n \geq n_{1} \in \mathbb{N}\left(n_{0}\right)$. Let $z_{n}$ be defined by (5). Then $z_{n}>0$ for $n \geq n_{1}$ and $\left\{z_{n}\right\}$ is unbounded and

$$
\Delta\left(a_{n}\left(\Delta z_{n}\right)^{\alpha}\right) \geq 0 .
$$

Thus $\left\{\Delta z_{n}\right\}$ is of constant sign and $\Delta z_{n}>0$ for all $n \geq n_{2} \in \mathbb{N}\left(n_{1}\right)$ since $\left\{z_{n}\right\}$ is unbounded. From (5) and inview of $\left\{z_{n}\right\}$ is increasing we have

$$
y_{n+l} \geq(1-p) z_{n+l}
$$

for $n \geq n_{2}$. From equation (1) and (18), we obtain

$$
\Delta\left(a_{n}\left(\Delta z_{n}\right)^{\alpha}\right) \geq q_{n} M(1-p)^{\alpha} z_{n+l}^{\alpha}, \quad n \geq n_{2} .
$$

Now summing (19) from $n$ to $s-1$, we have

$$
a_{s}\left(\Delta z_{s}\right)^{\alpha}-a_{n}\left(\Delta z_{n}\right)^{\alpha} \geq M(1-p)^{\alpha} \sum_{t=n}^{s-1} q_{t} z_{s+l}^{\alpha} \geq M(1-p)^{\alpha} z_{n+l}^{\alpha} \sum_{t=n}^{s-1} q_{t}
$$

or

$$
\Delta z_{s} \geq M^{\frac{1}{\alpha}}(1-p) z_{n+l}\left(\frac{1}{a_{s}} \sum_{t=n}^{s-1} q_{t}\right)^{\frac{1}{\alpha}}
$$

Again summing the last inequality in $s$ from $n$ to $n+l-1$, we obtain

$$
z_{n+l}-z_{n} \geq M^{\frac{1}{\alpha}}(1-p) z_{n+l} \sum_{s=n}^{n+l-1}\left(\frac{1}{a_{s}} \sum_{t=n}^{s-1} q_{t}\right)^{\frac{1}{\alpha}} .
$$


Hence

$$
z_{n+l}\left(M^{\frac{1}{\alpha}}(1-p) \sum_{s=n}^{n+l-1}\left(\frac{1}{a_{s}} \sum_{t=n}^{s-1} q_{t}\right)^{\frac{1}{\alpha}}-1\right) \leq 0
$$

which contradicts condition (17). This completes the proof of the theorem.

Remark 5. For difference equation without neutral term $(p=0)$ Theorem 6 provides the result of Wong and Agarwal [12].

Example 4. Consider the following difference equation

$$
\Delta\left(\frac{1}{(2 n+3)^{3}}\left(\Delta\left(y_{n}+\frac{1}{2} y_{n+1}\right)\right)^{3}\right)=\frac{1}{4(n-3)^{3}} y_{n+3}^{3}, \quad n \geq 4
$$

All conditions of Theorem 6 are satisfied and hence every unbounded solution of equation (21) oscillates. In fact $\left\{y_{n}\right\}=\left\{n(-1)^{n}\right\}$ is such a solution of equation (21).

Next we consider the case $\alpha=1$ and $a_{n} \equiv 1$ in equation (1) and discuss the oscillation of all unbounded solutions of equation (1) by relaxing the condition (16).

Theorem 7. With respect to the difference equation (1) assume that $p>0$. If

$$
\lim _{n \rightarrow \infty} \sup \sum_{s=n}^{n+l-1}(n+l-s) q_{s}^{*}>\frac{1+p}{M}
$$

where $q_{n}^{*}=\min \left\{q_{n}, q_{n-k}\right\}$, then every unbounded solution of equation (1) is oscillatory.

Proof. Assume without loss of generality that equation (1) has an eventually positive unbounded solution $\left\{y_{n}\right\}$. Then $z_{n}>0$ for $n \geq n_{1} \in \mathbb{N}\left(n_{0}+k\right),\left\{z_{n}\right\}$ is unbounded and from equation (1), we have

$$
\Delta^{2} z_{n} \geq M g_{n} y_{n+l}
$$

Thus $\Delta^{2} z_{n} \geq 0$ and this implies that $\Delta z_{n}$ is of constant sign. But if we take $\Delta z_{n}<0$, then $\left\{z_{n}\right\}$ would be bounded. Therefore $\Delta z_{n}>0$ for $n \geq n_{2} \in \mathbb{N}\left(n_{1}\right)$. Let

$$
x_{n}=z_{n}+p z_{n-k}
$$

then $x_{n}>0$ and $\Delta x_{n}>0$ for all large $n$ and further

$$
\begin{aligned}
\Delta^{2} x_{n} & \geq \Delta^{2} z_{n}+p \Delta^{2} z_{n-k} \\
& \geq M q_{n} y_{n+l}+p M q_{n-k} y_{n+l-k} \\
& \geq M q_{n}^{*}\left(y_{n+l}+p y_{n+l-k}\right) \\
& \geq M q_{n}^{*} z_{n+l} \\
& \geq \frac{M q_{n}^{*}}{(1+p)} x_{n+l} .
\end{aligned}
$$


Hence $\left\{x_{n}\right\}$ is a positive solution of the inequality

$$
\Delta^{2} x_{n}-\frac{M q_{n}^{*}}{1+p} x_{n+l} \geq 0
$$

for $n \geq n_{2}$. Summing (23) from $n$ to $j-1$, we have

$$
\Delta x_{j}-\Delta x_{n} \geq \frac{M}{1+p} \sum_{s=n}^{j-1} q_{s}^{*} x_{s+l}
$$

Now summing in $j$ from $n$ to $n+l-1$, we are led to

$$
x_{n+l}-x_{n}-\Delta x_{n}(l) \geq \frac{M}{1+p} \sum_{s=n}^{n+l-1}(n+l-s) q_{s}^{*} x_{s+l} .
$$

Consequently, using the monotonicity of $\left\{x_{n}\right\}$, we obtain

$$
x_{n+l}\left[\frac{M}{1+p} \sum_{s=n}^{n+l-1} q_{s}^{*}(n+l-s)-1\right] \leq 0
$$

which contradicts condition (22). The proof is now complete.

In our next theorem we consider the case when the condition (22) is not satisfied.

Theorem 8. With respect to the difference equation (1) assume that $p>0$. Further assume that the exists a sequence $\left\{b_{n}\right\}$ and a positive integer such that

$$
\begin{gathered}
b_{n}>0, \quad \Delta b_{n} \leq 0, \quad n \in \mathbb{N}\left(n_{0}\right) \\
l \geq 2 m
\end{gathered}
$$

and

$$
q_{n}^{*} \geq \frac{1+p}{M} b_{n+1} b_{n+m} \geq 0
$$

where $q_{n}^{*}$ is the same as defined in Theorem 8. If the difference inequality

$$
\Delta u_{n}-b_{n+m} u_{n+m} \geq 0
$$

has no eventually positive solution then all unbounded solutions of equation (1) are oscillatory.

Proof. Assume that $y_{n}>0$ is an unbounded solution of equation (1). Let $z_{n}$ and $x_{n}$ be the same as defined in Theorem 8 . Then proceeding exactly as in the proof of Theorem 8, we obtain (23). We put

$$
d_{n}=\Delta x_{n}+b_{n} x_{n+m}
$$


then $\left\{d_{n}\right\}$ is positive and

$$
\Delta d_{n}-b_{n+1} d_{n+m}=\Delta^{2} x_{n}+\Delta b_{n} x_{n+m}-b_{n+1} b_{n+m} x_{n+2 m} .
$$

Hence, in view of (23)-(26) one gets for all large $n$.

$$
\begin{aligned}
\Delta d_{n}-\frac{\Delta b_{n}}{b_{n}} d_{n}-b_{n+1} d_{n+m} & \geq \Delta^{2} x_{n}-\frac{\Delta b_{n}}{b_{n}} \Delta z_{n}-b_{n+1} b_{n+m} x_{n+2 m} \\
& \geq \Delta^{2} x_{n}-b_{n+1} b_{n+m} x_{n+2 m} \\
& \geq \Delta^{2} x_{n}-\frac{M q_{n}^{*}}{1+p} x_{n+l} \geq 0
\end{aligned}
$$

Setting

$$
d_{n}=b_{n} v_{n}
$$

we conclude that $\left\{v_{n}\right\}$ is a positive solutions of (27). This contradiction completes the proof.

Corollary 1. With respect to the difference equation (1) assume that $p>0$. Moreover assume that conditions (23)-(26) hold.

If

$$
\lim _{n \rightarrow \infty} \sup \left[\frac{1}{m} \sum_{s=n}^{n+m} b_{n+m}\right]>\frac{m^{m}}{(m+1)^{m+1}}
$$

then equation (1) does not allow unbounded non-oscillatory solution.

Proof. It is known that [5] condition (28) is sufficient for (27) to have no eventually positive solutions. Hence the assertion follows from Theorem 8.

We conclude this paper with the following remarks.

Remark 6. Theorem 8 permits to apply any sufficient condition for absence of eventually positive solution of (27) to obtain unbounded oscillation criteria for equation (1) provided that conditions (23)-(26) hold.

\section{References}

[1] R. P. Agarwal, Difference Equations and Inequalities, Second Edition, Marcel Dekker, New York, 2000.

[2] R. P. Agarwal and P.J.Y.Wong, Advanced Topics in Difference Equations, Kluwer publication Dordrecht, 1997.

[3] R. P. Agarwal, S. R. Grace and D. O' Regan, On the oscillation of certain second order difference equations, J. Diff. Eqns. Appl. 9(2003), 109-119.

[4] D. D. bainow and D. P. Mishev, Oscillation Theory for Neutral Equations with Delay, Adam Hilger, 1991.

[5] L. Györi and G. Ladas, Oscillation Theory of Delay Differential Equations, Clarendar Press, New York, 1991. 
[6] B. S. Lalli and B. G. Zhang, On the existsence of positive solutions and bounded oscillations for neutral difference equations, J. Math. Anal. Appl. 166(1992), 272-287.

[7] P. Sundaram and E. Thandapani, Oscillation and nonoscillation theorems for second order quasilinear functional difference equations, Indian J. Pure App. Math. 31(2000), 37-47.

[8] E. Thandapani, J. R. Graef and P. W. Spikes, On the oscillation of solutions of second order quasilinear difference equations, Nonlinear world 3(1996), 545-565.

[9] E. Thandapani, S. Pandian and R. K. Balasubramanian, Asymptotic behaviour of solutions of a class of second order quasilinear difference equations, Kyung. Math. J. (to appear).

[10] E. Thandapani, Asymptotic and oscillatory behaviour of solutions of second order nonlinear delay difference equations, Riv. Math. Univ. Parma 1(1992), 105-113.

[11] E. Thandapani, R. Arul and P. S. Raja, Bounded oscillation of second order unstable neutral type difference equation, J. Appl. Math. Computing (to appear).

[12] P. J. Y. Wong and R. P. Agarwal, Oscillation and nonoscillation of half-linear difference equation generated by deviating arguments, Comput. Math. Appl. 36(1998), 11-26.

Department of Mathematics, Peryiar University, Salem-636011, TamilNadu, India.

Department of Mathematcs, Dr. Ambedkar Government arts College, Chennai-600039, India. 